\title{
Minimum Inhibitory Concentration and Susceptibility Patterns of Organisms to Fosfomycin as Determined by BD Phoenix M50
}

\author{
Jacob Birdsall $^{1 *}$, Steven Siarakas ${ }^{1,2}$ and John Merlino ${ }^{1,2}$ \\ ${ }^{1}$ Department of Microbiology and Infectious Diseases, Concord Repatriation \\ General Hospital, NSW Health Pathology, Australia \\ ${ }^{2}$ Department of Immunology and Infectious Diseases, Central Clinical School, \\ Faculty of Medicine and Health, University of Sydney, Australia
}

*Corresponding Author: Jacob Birdsall, Department of Microbiology and Infectious Diseases, Concord Repatriation General Hospital, NSW Health Pathology, Australia.
Received: March 03, 2021

Published: April 07, 2021

(C) All rights are reserved by Jacob Birdsall., et al.

\begin{abstract}
Objectives: To compare the fosfomycin minimum inhibitory concentrations of Enterobacterales and non-Enterobacterales, as determined by the BD Phoenix M50, to EUCAST and CLSI clinical breakpoints.

Methods: 158 Gram-negative organisms isolated from clinical samples underwent susceptibility testing using the BD Phoenix M50, NMIC-404 panel. The fosfomycin MICs of both Enterobacterales and non-Enterobacterales were compared to EUCAST and CLSI clinical breakpoints.

Results: A total of 138 Enterobacterales were tested, including 81 E. coli isolates. 131 (95.0\%) were considered susceptible using EUCAST breakpoints and 134 (97.1\%) were considered susceptible using CLSI breakpoints. Of the 20 non-Enterobacterales tested, 5 isolates (25.0\%) showed an MIC below $\leq 32 \mathrm{mg} / \mathrm{L}$ (EUCAST breakpoint for Enterobacterales), and 16 isolates (80.0\%) had an MIC of $\leq 64 \mathrm{mg} / \mathrm{L}$ (CLSI breakpoint for E. coli isolates).

Conclusions: This study showed high susceptibility rates of most Enterobacterales to fosfomycin, even in the presence of resistance mechanisms (ESBL and CPO), while most of the non-Enterobacterales tested showed elevated MICs to fosfomycin.
\end{abstract}

Keywords: E. coli; Enterobacterales; EUCAST; Fosfomycin

\section{Introduction}

Fosfomycin is a broad-spectrum antibiotic commonly used in UTIs. It has activity against most Enterobacterales, notably Escherichia coli, Proteus mirabilis and Citrobacter spp., while more varied activity has been described against non-Enterobacterales [1]. Mowlaboccus., et al. performed a study which showed low incidence of fosfomycin resistance in Escherichia coli urinary tract infection isolates in Australia (2 of 1033 isolates resistant) [2].

Fosfomycin acts by inhibiting the MurA enzyme, which catalyses the first stage in peptidoglycan synthesis [3]. EUCAST clinical breakpoints for Enterobacterales in uncomplicated urinary tract infection (UTI), are MIC of $\leq 32 \mathrm{mg} / \mathrm{L}$ (susceptible) and MIC of $>32$ $\mathrm{mg} / \mathrm{L}$ (resistant), with no other MIC breakpoints or zone diameters set for non-Enterobacterales [4,5]. CLSI clinical breakpoints, which apply only to $E$. coli urinary tract isolates, are MIC of $\leq 64 \mathrm{mg} / \mathrm{L}$ (susceptible); MIC of $128 \mathrm{mg} / \mathrm{L}$ (intermediate); MIC of $\geq 256 \mathrm{mg} / \mathrm{L}$ (resistant) [8].

A previous study examined the accuracy of the Phoenix in detecting fosfomycin resistance compared to other methods (i.e. disc 
diffusion, Etest, Vitek). Despite the current gold standard for susceptibility testing being agar dilution and disc diffusion, the Phoenix showed $99.5 \%$ categorical agreement for E. coli, and $93 \%$ for K. pneumoniae, with significantly lower major/very major errors compared to the other methods [7].

\section{Aim of the Study}

This study was designed to compare the fosfomycin MICs of different Enterobacterales and non-Enterobacterales (consisting of Pseudomonas spp.), as determined by the BD Phoenix M50, NMIC404 panel, to EUCAST and CLSI clinical breakpoints.

\section{Methods}

Over a three-month period, a total of 158 Gram-negative organisms isolated from clinical samples, were collected. This included 23 extended-spectrum $\beta$-lactamase (ESBL) producing organisms and 5 carbapenemase-producing organisms (CPO) (IMP4: $\mathrm{n}=2$, KPC: $n=1$, oxa48: $n=1$, AIM: $n=1$ ). Each isolate had susceptibility testing performed on a pure culture using the BD Phoenix M50, NMIC-404 panel. This data was correlated and fosfomycin MICs of both Enterobacterales and non-Enterobacterales were compared to EUCAST and CLSI clinical breakpoints.

\section{Results and Discussion}

A total of 138 Enterobacterales were tested, 81 of which were $E$. coli. Of the E. coli isolates, 81 (100.0\%) were classified as susceptible, including 18 ESBL positive isolates, when EUCAST and CLSI breakpoints were used.

Of the other Enterobacterales ( $\mathrm{n}=57), 50(87.7 \%)$ were classified as susceptible (MIC of $\leq 32 \mathrm{mg} / \mathrm{L}$ ) including 5 ESBL producing organisms and $4 \mathrm{CPO}$, while 7 (12.3\%) were classified as resistant using EUCAST breakpoints (MIC of $>32 \mathrm{mg} / \mathrm{L}$ ); the majority of these were Morganella morganii. Using CLSI breakpoints, 53 isolates $(93.0 \%)$ were classified as susceptible (MIC of $\leq 64 \mathrm{mg} / \mathrm{L}$ ), while 4 isolates (7.0\%) showed an MIC of $>64 \mathrm{mg} / \mathrm{L}$, which were classified as either intermediate (MIC of $128 \mathrm{mg} / \mathrm{L}$ ) or resistant (MIC of $\geq 256 \mu \mathrm{g} / \mathrm{mL}$ ); all of which were M. morganii (Figure 1 ).

A total of 20 non-Enterobacterales were tested, comprised of Pseudomonas aeruginosa $(\mathrm{n}=19)$ and Pseudomonas putida $(\mathrm{n}=1)$. Using EUCAST breakpoints set for Enterobacterales, only 5 isolates (25.0\%) showed an MIC below the breakpoint of $\leq 32 \mathrm{mg} / \mathrm{L}$, while 15 isolates (75.0\%) had an MIC > $32 \mathrm{mg} / \mathrm{L}$. Using CLSI breakpoints,

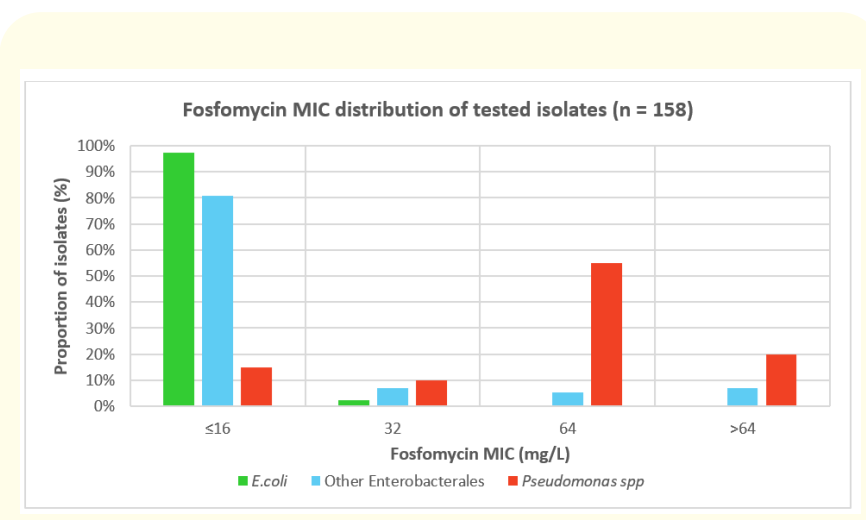

Figure 1: Fosfomycin MIC distribution of tested isolates.

16 isolates (80.0\%) had an MIC of $\leq 64 \mathrm{mg} / \mathrm{L}$, including $1 \mathrm{CPO}$, while 4 isolates $(20.0 \%)$ had an MIC $>64 \mathrm{mg} / \mathrm{L}$ (Figure 1).

Of the 23 ESBL producing organisms tested, 23 (100.0\%) had an MIC of $\leq 32 \mathrm{mg} / \mathrm{L}$, classifying them as sensitive using both EUCAST and CLSI, with 22 (95.6\%) having an MIC of $\leq 16 \mathrm{mg} / \mathrm{L}$. Of the 4 CPO positive Enterobacterales, 4 (100.0\%) had an MIC $\leq 32$ $\mathrm{mg} / \mathrm{L}$ classifying them as susceptible according to EUCAST and CLSI (Table 1).

Although $100 \%$ of the Klebsiella isolates which were tested had an MIC of $\leq 32 \mathrm{mg} / \mathrm{L}$, indicating in vitro susceptibility, reduced clinical activity, emergence of resistance and failure in treatment has been shown to be significant among Klebsiella spp [6,7].

\section{Conclusion}

The results of this study showed high susceptibility rates of most Enterobacterales to fosfomycin (excluding Morganella spp.), even in the presence of presumed resistance mechanisms, while most of the Pseudomonas spp. tested showed elevated MICs to fosfomycin. EUCAST and CLSI have not set clinical breakpoints for Pseudomonas spp. Fosfomycin may be a useful therapeutic option for most Enterobacterales, including ESBL and CPO positive isolates, however further study is required to ensure adequate clinical activity. Fosfomycin is unlikely to be beneficial in the treatment of non-Enterobacterales.

\section{Transparency Declarations}

None to declare. 


\begin{tabular}{|c|c|c|c|c|c|c|c|}
\hline & \multirow{2}{*}{\multicolumn{2}{|c|}{ Organism Name/Genus }} & \multirow{2}{*}{$\mathbf{n}$} & \multicolumn{4}{|c|}{ Fosfomycin MIC (mg/L) } \\
\hline & & & & $\leq 16$ & 32 & 64 & $>64$ \\
\hline \multirow{12}{*}{ Enterobacterales } & \multicolumn{2}{|c|}{ Enterobacter spp. } & 5 & $\begin{array}{c}5 \\
\text { E. cloacae (ESBL: } \\
\mathrm{n}=2, \text { IMP4: } \mathrm{n}=1)\end{array}$ & 0 & 0 & 0 \\
\hline & \multicolumn{2}{|c|}{ E. coli } & 81 & $\begin{array}{c}79 \\
(\text { ESBL: } n=18)\end{array}$ & 2 & 0 & 0 \\
\hline & \multicolumn{2}{|c|}{ Klebsiella spp. } & 22 & $\begin{array}{c}21 \\
\text { K. pneumoniae } \\
\text { (oxa48: } \mathrm{n}=1 \text {, ESBL: } \\
\mathrm{n}=1 \text { ) }\end{array}$ & $\begin{array}{l}1 \\
\text { K. pneumoniae } \\
\text { (KPC+ESBL: } \mathrm{n} \\
=1 \text { ) }\end{array}$ & 0 & 0 \\
\hline & \multicolumn{2}{|c|}{ Morganella spp. } & 4 & 0 & 0 & 0 & 4 \\
\hline & \multirow{2}{*}{ Proteus spp. } & P. mirabilis & 9 & 8 & 0 & 1 & 0 \\
\hline & & Other & 2 & 2 & 0 & 0 & 0 \\
\hline & \multicolumn{2}{|c|}{ Providencia spp. } & 2 & 0 & 0 & 0 & 0 \\
\hline & \multicolumn{2}{|c|}{ Raoutella spp. } & 1 & 0 & 0 & 1 & 0 \\
\hline & \multicolumn{2}{|c|}{ Salmonella spp. } & 3 & $\begin{array}{c}3 \\
\text { Salmonella spp. } \\
(\text { ESBL: } \mathrm{n}=1)\end{array}$ & 0 & 0 & 0 \\
\hline & \multicolumn{2}{|c|}{ Serratia spp. } & 3 & 0 & 3 & 0 & 0 \\
\hline & \multicolumn{2}{|c|}{ Shigella spp. } & 1 & 1 & 0 & 0 & 0 \\
\hline & \multicolumn{2}{|c|}{ Total } & 138 & 125 & 6 & 3 & 4 \\
\hline \multirow{3}{*}{ Non-enterobacterales } & \multirow{2}{*}{$\begin{array}{l}\text { Pseudomonas } \\
\text { spp. }\end{array}$} & P. aeruginosa & 19 & 3 & 2 & $\begin{array}{c}11 \\
(\text { AIM: } n=1)\end{array}$ & 3 \\
\hline & & Other & 1 & 0 & 0 & 0 & 1 \\
\hline & \multicolumn{2}{|c|}{ Total } & 20 & 3 & 2 & 11 & 4 \\
\hline
\end{tabular}

Table 1: Breakdown of tested isolates and respective fosfomycin MIC distribution.

\section{Conflicts of Interest and Sources of Funding}

The authors state that there are no conflicts of interest to disclose. No funding was received for this study.

\section{Bibliography}

1. Therapeutic Goods Administration: Australian Public Assessment Report for Fosfomycin (2018).

2. Mowlaboccus S., et al. "Identification and characterisation of fosfomycin resistance in Escherichia coli urinary tract infection isolates from Australia”. International Journal of Antimicrobial Agents 56.4(2020):106121.

3. Jin-Yi Zhu., et al. "Functional Consequence of Covalent Reaction of Phosphoenolpyruvate with UDP-Nacetylglucosamine 1-Carboxyvinyltransferase (MurA)". Journal of Biological Chemistry 287.16 (2012): 12657-12667.

4. EUCAST Clinical Breakpoint tables for interpretation of MICs and zone diameters Version 9.0 (2019).

5. Fosfomycin: Rationale for the EUCAST clinical breakpoints Version 1.0 (2013). 
6. Iain J Abbott., et al. "Fosfomycin efficacy and emergence of resistance among Enterobacteriaceae in an in vitro dynamic bladder infection model". Journal of Antimicrobial Chemotherapy 73.3 (2018): 709-719.

7. Johan W Mouton. "Challenging drugs for AST: Fosfomycin”. ESCMID (2019).

8. CLSI. Performance Standards for Antimicrobial Susceptibility Testing. $30^{\text {th }}$ edition. CLSI supplement M100. Wayne, PA: Clinical and Laboratory Standards Institute (2020).

\section{Assets from publication with us}

- Prompt Acknowledgement after receiving the article

- Thorough Double blinded peer review

- Rapid Publication

- Issue of Publication Certificate

- High visibility of your Published work

Website: www.actascientific.com/

Submit Article: www.actascientific.com/submission.php

Email us: editor@actascientific.com

Contact us: +919182824667 